\title{
Purtscher-Like Retinopathy as First Presentation in a Newly Diagnosed Systemic Lupus Erythematosus Patient and Its Response to Treatment
}

\author{
Ng Chun Ruh', She Poh Fong², Ong Ming Jew ${ }^{2}$, Loh Yet Lin'1 \\ ${ }^{1}$ Unit Rheumatology, Department of Medicine, Hospital Sultan Ismail, Johor Bahru, Johor, Malaysia \\ ${ }^{2}$ Department of Ophthalmology, Hospital Sultan Ismail, Johor Bahru, Johor, Malaysia \\ Email: angiepaeds@gmail.com
}

How to cite this paper: Ruh, N.C., Fong, S.P., Jew, O.M. and Lin, L.Y. (2021) Purtscher-Like Retinopathy as First Presentation in a Newly Diagnosed Systemic Lupus Erythematosus Patient and Its Response to Treatment. Open Access Library Journal, 8: e7485. https://doi.org/10.4236/oalib.1107485

Received: April 30, 2021

Accepted: July 4, 2021

Published: July 7, 2021

Copyright $\odot 2021$ by author(s) and Open Access Library Inc.

This work is licensed under the Creative Commons Attribution International License (CC BY 4.0).

http://creativecommons.org/licenses/by/4.0/

\begin{abstract}
Impaired visual acuity was the first presentation in a 32-year-old lady who was subsequently diagnosed as systemic lupus erythematosus (SLE) at 6 months post delivery. We report a rare case of Purtscher-like retinopathy in a SLE patient, its clinical presentation, funduscopic findings and response to steroid, panretinal photocoagulation and mycophenolate mofetil (MMF).
\end{abstract}

\section{Subject Areas \\ Rheumatology}

\section{Keywords}

SLE, Purtscher-Like Retinopathy, Visual Acuity, Mycophenolate Mofetil

\section{Introduction}

Purtscher retinopathy was first described by an Austrian ophthalmologist, Dr Othmar Purtscher in 1910 in a man with severe head trauma. [1] The diagnosis of the condition is made on clinical grounds with the presentation of sudden vision loss occurring hours to days following the causal event with characteristic funduscopic findings of Purtscher flecken in 50\% of cases which is pathognomonic of the disease. [2] Subsequently similar retinal abnormalities had been observed in other types of injury primarily in chest compression and systemic conditions which included acute pancreatitis, SLE, thrombotic thrombocytopenic purpura among many others. In diseases of nontraumatic etiology with typical 
retinal findings the term used is Purtscher-like retinopathy. Agrawal et al. reported in his study that out of 15 patients all were symptomatic, $60 \%$ had loss of both visual acuity and visual field, 33\% loss of visual acuity alone and 7\% had only visual field impairment. Furthermore, $60 \%$ had bilateral whereas $40 \%$ had unilateral eye involvement. [3] Its pathogenesis remains unclear. The most accepted mechanism is embolisation of complement mediated leucocyte aggregates, fat, air, fibrin or platelet depending on the underlying pathology leading to occlusion of precapillary arterioles and infarction of microvascular bed. [4] There is no evidence based guideline on the management of Purtscher-like retinopathy. Controlling the underlying disease and its activity seems a logical approach in its treatment. There is paucity of data in the literature on SLE and Purtscher-like retinopathy and its management. Here we would like to report a case of a SLE patient who presented with Purtscher-like retinopathy at diagnosis and its response to corticosteroid and MMF.

\section{Case Report}

A previously well 32-year-old Malay housewife was admitted to our hospital when she presented at the emergency department with 4 months' history of facial rash. The patient was 6 months postpartum. The rash initially started on the left side of her face and subsequently spread and involved the rest of it, both ears, scalp, trunk and feet. The rash was mildly pruritic. She also had photosensitivity, hair loss, oral ulcers and arthralgia. She lost about $10 \mathrm{~kg}$ since the onset of her illness and had poor oral intake due to painful oral ulcers.

She also complained of blurring of vision for 1 month. It was insidious in onset and progressively worsening. The right eye was more affected than the left.

Besides that, she had intermittent fever for 2 weeks and lethargy. This was associated with non-productive cough for 1 week.

She has 3 living children with an uneventful antenatal history.

There was no significant family history.

She does not smoke or consume alcohol.

Clinical examination revealed a medium build lady. She had tachycardia with a heart rate of $134 \mathrm{bpm}$. Her blood pressure was 101/64 mmhg and she was febrile with a temperature of $39.3^{\circ} \mathrm{C}$. She had well demarcated, hyperpigmented, dry, crusted and scaly plaques over her whole face, scalp, bilateral ears, back and anterior chest. She also had vasculitic purpuric rash over her palms and toes. She had oral ulcers and her hair was sparse.

The patient's cardiovascular and respiratory systems and abdominal examinations revealed no abnormality. Her central nervous system was normal except for the second cranial nerve. Musculoskeletal examination revealed no active synovitis.

She was referred to the ophthalmologist for visual assessment. Ophthalmic examination showed a visual acuity of light perception in the right eye and counting finger in the left eye. Both her pupils were equal and reactive. There was no 
relative afferent pupillary defect. Both corneas showed presence of punctate epithelial erosions and her schirmer test was positive at $0 \mathrm{~mm}$ each. Both lenses appeared clear. Intraocular pressure in both eyes were within the normal range. Funduscopy of both eyes revealed presence of pale optic discs with ghost vessels and multiple cotton wool spots. There was presence of typical Purtscher flecken in the posterior poles bilaterally (Figure $1 \&$ Figure 2). Optical coherence tomography (OCT) was done which showed both eyes had cystoid macular oedema (Figure 3).

Unfortunately, fundus fluorescein angiography was not performed as patient was not keen for the invasive procedure.

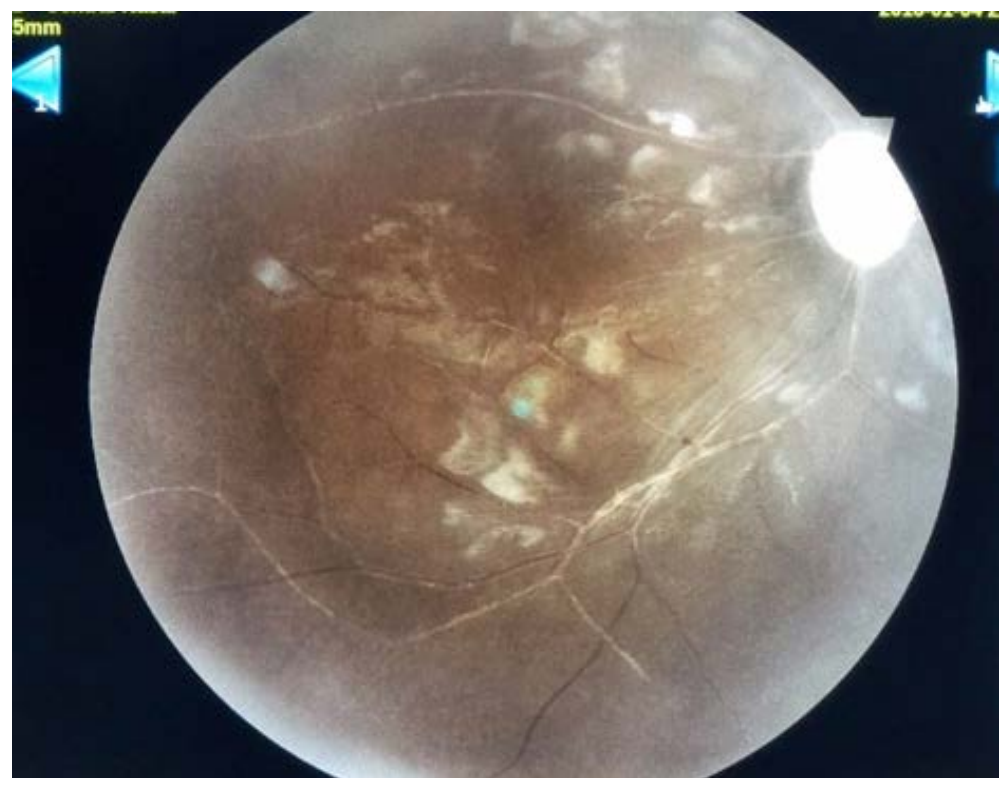

Figure 1. Right eye funduscopic findings.

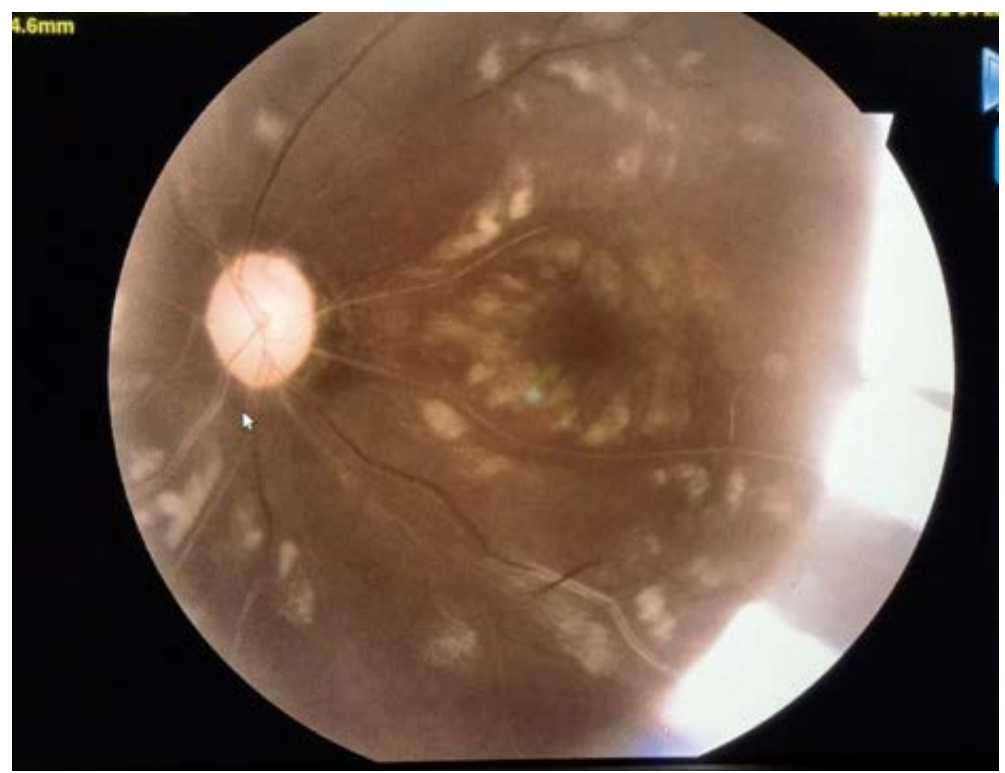

Figure 2. Left eye funduscopic findings. 

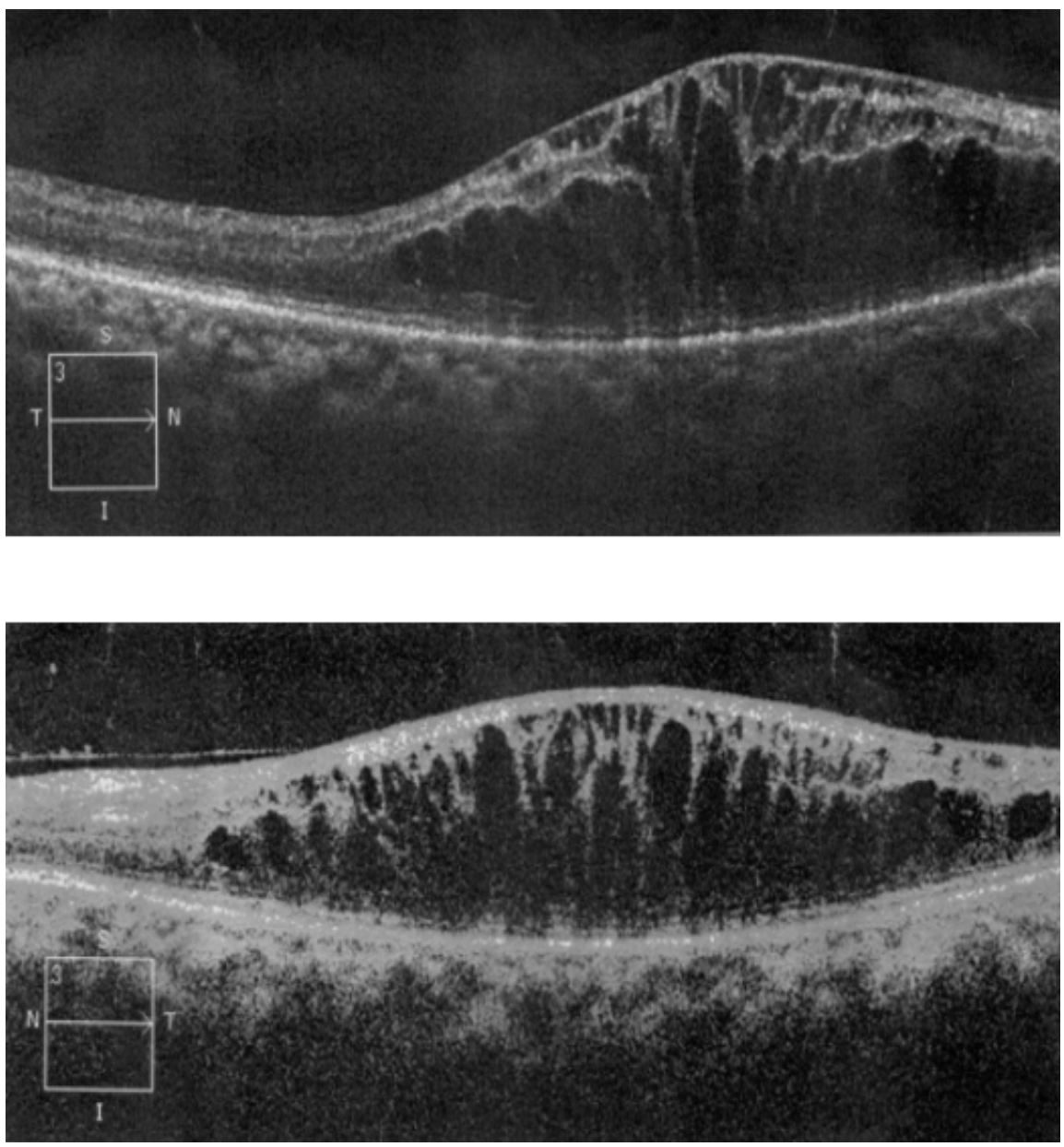

Figure 3. Right (top) and left eye (bottom) OCT showed cystoid macular oedema (right side worse than left).

Laboratory analysis showed presence of pancytopenia in the full blood count with a haemoglobin of $9.4 \mathrm{~g} / \mathrm{dl}(12.0-15.0 \mathrm{~g} / \mathrm{dl})$, white blood cell count of 3200 cells/ul (4000 - 11,000 cells/ul) of which $39.2 \%(20 \%-40 \%)$ were lymphocytes and platelet of $86,000 / \mathrm{ul}(150,000-400,000 / \mathrm{ul})$. Direct coombs' test was positive. However there was no haemolysis in the peripheral blood film and the reticulocyte count was normal. Inflammatory markers erythrocyte sedimentation rate (ESR) and c-reactive protein (CRP) were $42 \mathrm{~mm} / \mathrm{h}$ and $3 \mathrm{mg} / \mathrm{L}$ respectively. Renal profile was normal but there was presence of $1+$ protein in the urine examination. 24-hour urine protein was $0.47 \mathrm{~g} /$ day. Total protein was $79 \mathrm{~g} / \mathrm{L}$ and serum albumin and globulin levels were $27 \mathrm{~g} / \mathrm{L}$ and $52 \mathrm{~g} / \mathrm{L}$ respectively. Serum alanine transaminase was $30 \mathrm{u} / \mathrm{l}$ and aspartate transaminase was $95 \mathrm{u} / \mathrm{l}$. Antinuclear antibody (ANA) test was positive at a titre of 1:1280 and with speckled pattern. Anti Smith antibody and anti SSA antibody were both positive at a titre of $>600$ $\mathrm{U} / \mathrm{ml}$ each. Complement 3 was low $(0.59 \mathrm{~g} / \mathrm{L})$ but complement 4 was normal $(0.224 \mathrm{~g} / \mathrm{L})$.The patient's thyroid function test was consistent with hypothyroidism as serum thyroid stimulating hormone and T4 were $6.75 \mathrm{mIU} / \mathrm{L}$ and 8.9 pmol/L respectively. Thrombophilia screening was negative. Blood and urine 
culture were negative.

Chest X-ray was normal and 12 lead electrocardiogram showed presence of sinus tachycardia with T inversion in leads I, II, III, V4, V5 and V6.

The patient was diagnosed as SLE with constitutional, mucocutaneous, renal and haematological manifestations and bilateral Purtscher-like retinopathy, secondary Sjogren's syndrome and hypothyroidism.

She was started on intravenous methylprednisolone $500 \mathrm{mg}$ daily for 3 days followed by intravenous hydrocortisone $100 \mathrm{mg}$ three times a day. She was also commenced on L-thyroxine supplement for hypothyroidism. Cyclophosphamide was discussed as a possible treatment option with the patient. However she was not keen.

The patient's stay was complicated by methicillin-sensitive staphylococcus aureus bacteraemia with pneumonia secondary to right forearm thrombophlebitis 1 week after admission. She was empirically started on intravenous cloxacillin and cefepime prior to the confirmation of the blood culture results. She became afebrile after 2 days of antibiotics. Concomitantly she was also receiving intravenous hydrocortisone. The patient developed confusion with disorientation to time and was noted to be talking to herself 4 days later. There was no trauma or metabolic derangement and her blood pressure was normal. Contrast enhanced computed tomography of the brain which was done was normal. Lumbar puncture findings included presence of colourless cerebrospinal fluid (CSF) with an opening pressure of $12 \mathrm{~cm} \mathrm{H}_{2} \mathrm{O}$, protein level of $0.53 \mathrm{~g} / \mathrm{L}(0.2-0.5 \mathrm{~g} / \mathrm{L})$, glucose of $2.7 \mathrm{mmol} / \mathrm{L}(2.5-4.4 \mathrm{mmol} / \mathrm{L})$ and negative results for cell count, gram stain and culture. The random blood sugar was $5.0 \mathrm{mmol} / \mathrm{L}$.

MRI brain was not done. The abnormal behaviour resolved after discontinuation of cefepime 2 days later. However, cerebral lupus cannot be ruled out. She completed a 2-week course of intravenous cloxacillin. Echocardiogram done revealed presence of impaired left ventricular function with global hypokinesia with an ejection fraction of $33 \%$.

She was discharged after 1 month of hospitalisation with tab prednisolone 40 mg OD, tab hydroxychloroquine $200 \mathrm{mg}$ OD, tab L-thyroxine $50 \mathrm{mcg} \mathrm{OD}$, tab calcium carbonate $500 \mathrm{mg}$ OD daily, cap calcitriol $0.25 \mathrm{mcg}$ BD and sunblock.

The patient had a total of 5 sessions of panretinal photocoagulation done for each eye after detection of neovascularization and retinal haemorrhage. She was also given copious amount of ocular lubricants to treat her dry eyes.

During her follow-up 1 month later, there was no constitutional symptom, recurrence of abnormal behaviour or newly onset mucocutaneous lesions. Her right eye vision improved slightly to hand movement and left was 6/60. Laboratory investigations also showed a normal full blood count with a haemoglobin of 12.2 $\mathrm{g} / \mathrm{dl}$, white blood cell count of 10,600 cells/ul of which $37 \%$ were lymphocytes and platelet of 330,000/ul and her serum albumin level was $36 \mathrm{~g} / \mathrm{L}$. Urine examination was normal with nil protein and red blood cell. The patient was commenced on azathioprine as a steroid sparing agent of which she developed trans- 
aminitis 3 weeks later at the dose of $100 \mathrm{mg}$ OD. When the liver function test normalized 3 weeks after discontinuation of azathioprine, MMF was started replacing azathioprine at 5 months post discharge. The dose of MMF was titrated and maintained at $1 \mathrm{~g} \mathrm{BD}$ within a month without any adverse event.

Funduscopic findings 6 months after she was discharged revealed resolving Purtscher flecken and preretinal haemorrhage in both eyes with the presence of multiple laser marks in both eyes (Figure 4 and Figure 5). She was keeping well with no new rash or oral ulcer.

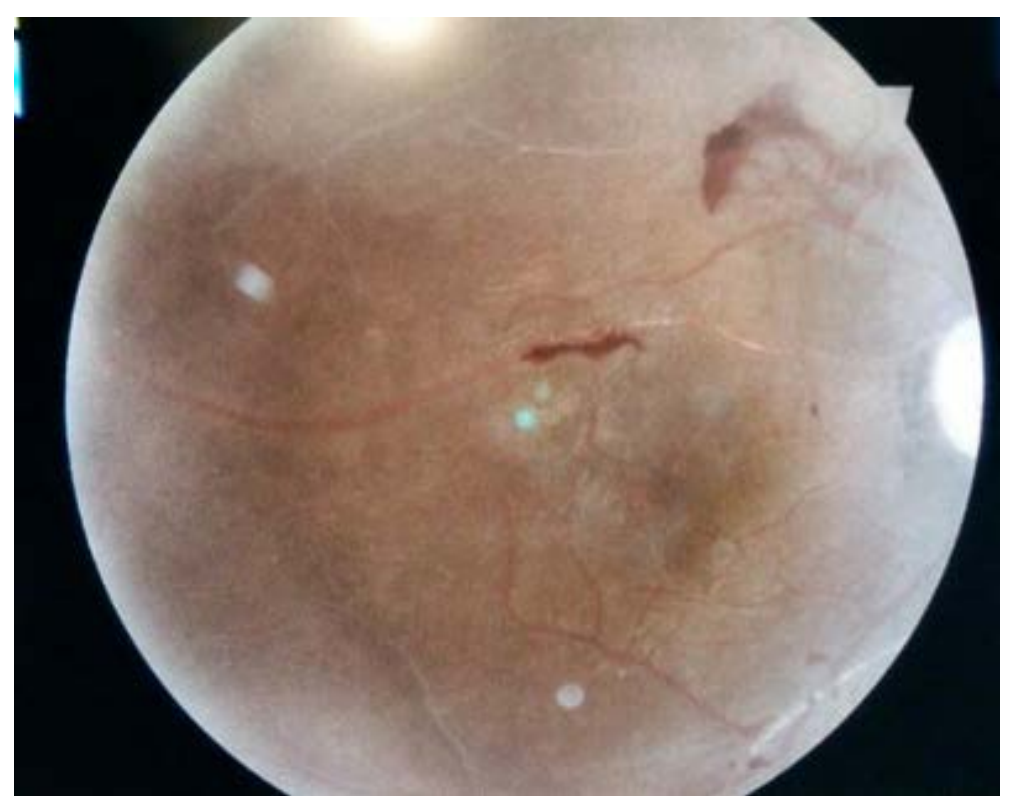

Figure 4. Right funduscopic findings.

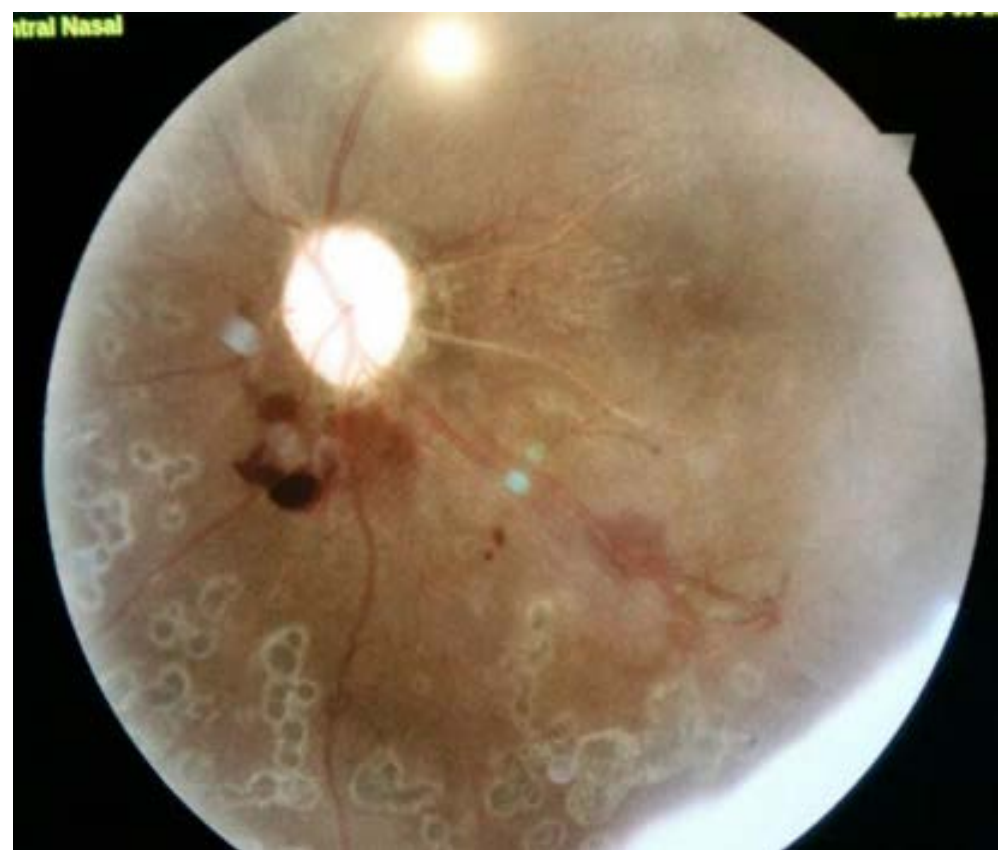

Figure 5. Left funduscopic findings. 
At 30 months of follow-up, her visual acuity remained the same with hand movement in her right eye and 6/60 in her left and repeated echocardiogram showed good left ventricular function with no regional wall movement abnormality and ejection fraction of $64.4 \%$. She was keeping well with no new vasculitic lesions seen. Her medications included tab prednisolone $10 \mathrm{mg} O \mathrm{OD}$, cap MMF $1 \mathrm{~g}$ BD, tab hydroxychloroquine $200 \mathrm{mg}$ OD, tab L-thyroxine $50 \mathrm{mcg}$ OD, tab calcium carbonate $500 \mathrm{mg}$ OD daily, cap calcitriol $0.25 \mathrm{mcg} \mathrm{BD}$, tab folic acid $5 \mathrm{mg}$ OD and sunblock.

\section{Discussion}

Purtscher-like retinopathy is a rare but severe ophthalmic complication of SLE. According to a study done by Agrawal et al., the yearly incidence of symptomatic Purtscher retinopathy and Purtscher-like retinopathy was reported as approximately 0.24 cases per million per year based on the UK population of 58.8 million (2001 census) and the Republic of Ireland population of 3.9 million (2002 census). [3]

In 2013 Miguel et al. proposed a diagnostic criteria for Purtscher-like retinopathy where at least 3 of the following 5 features should be fulfilled [2]:

1) Purtscher flecken,

2) Retinal haemorrhages in low to moderate number,

3) Cotton wool spots (confined to the posterior pole),

4) Probable explanatory etiology,

5) Complementary investigation compatible with diagnosis.

Our patient fulfilled four out of five criteria with SLE being the etiology. [2] She met the requirement of the 2019 EULAR/ACR classification criteria for SLE as she had positive ANA with a titre of 1:1280 with 3 clinical and 2 immunological criteria (fever, leucopenia, thrombocytopenia, non-scarring alopecia, oral ulcer, discoid lupus, low complement 3 and positive anti Smith antibody) with a total score of 19.

There were case reports which postulated the association between development of Purtscher-like retinopathy with pregnancy and childbirth as described by Blodi et al. and Olson et al. Some of the patients had preeclampsia (2 out of the 4 patients described by Blodi had preeclampsia and 1 had pancreatitis whereas the 1 patient reported by Olson had preeclampsia). [5] [6] Our patient had an uneventful obstetric history with normal vaginal delivery.

She developed confusion on day 13 of hospitalisation after receiving cefepime for 5 days for pneumonia. The acute episode resolved after discontinuation of cefepime. A case report published in 2016 illustrated cefepime induced encephalopathy in a 76 year old lady with normal renal function. [7] However the diagnosis of central nervous system (CNS) lupus cannot be totally ruled out. Furthermore correlation between CNS lupus and Purtscher-like retinopathy has been reported. An observational case series done by $\mathrm{Wu} \mathrm{C}$ et al. concluded that Purstscher-like retinopathy was associated with high disease activity with SLE- 
DAI-2K of more than 20 and 75\% of the patients had CNS manifestation. [8] The SLEDAI-2K in our patient would be 35 if she had CNS lupus.

There is no guideline on the treatment of Purtscher-like retinopathy but a reasonable therapeutic approach will be to manage the underlying disease. Our patient was given prompt immunosuppressive therapy with intravenous steroids and ocular treatment with panretinal photocoagulation. Subsequently hydroxychloroquine and MMF were added to treat the systemic activity of SLE. She was noted to have slight improvement in her vision after intravenous methylprednisolone. There was no further progress in her visual recovery despite MMF which was however administered some time after her initial manifestation. Intravenous cyclophosphamide was considered and discussed during the early part of her admission as she had severe visual impairment. However our patient declined and her stay was further complicated by bacteraemia. This delays the addition of an immunosuppressive agent. According to our local guideline, azathioprine can be used as a first line steroid sparing agent whereas MMF is second line. Unfortunately she developed complications with azathioprine and was eventually commenced on MMF.

Miguel et al. reported that good prognostic factors in Purtscher and Purtscher-like retinopathy included underlying etiologies such as pancreatitis and trauma, male gender and absence of macular oedema. [2] Our patient is a female with underlying SLE and had macular oedema which were not favourable prognostic factors. Most importantly her prognosis is poor due to late presentation with severe retinal changes and optic atrophy. Since then although she did not show much improvement in her visual acuity her vision remained stable. Early presentation could probably have prevented severe vision loss.

\section{Conclusion}

Ocular manifestations of SLE are common and maybe the initial presenting feature of the disease. This case report highlights the importance of recognition of rare eye condition such as Purtscher-like retinopathy in SLE patients by rheumatologists as it is sight-threatening. A combination of care by both the rheumatologist and ophthalmologist is of utmost importance to stabilise the disease with the aim of preserving ocular function and health. Steroid and MMF probably played a role in the stabilisation of her eye disease. At the same time, raising awareness of eye health and disease is a critical step to prevent irreversible vision loss.

\section{Consent}

Written informed consent was obtained from the patient for the publication of this case report and any accompanying images.

\section{Conflicts of Interest}

The authors declare no conflicts of interest. 


\section{References}

[1] Purtscher, O. (1910) Noch Unbekannte Befunde Nach Schadeltrauma. Bericht Uber Die Zusammenkunft Der Deutschen Ophthalmologischen Gesellschaft, 36, 294-301.

[2] Miguel, A.I.M., Henriques, F., Azevedo, L.F.R., et al. (2013) Systemic Review of Purtscher's and Purtscher-Like Retinopathies. Eye (Lond), 27, 1-13. https://doi.org/10.1038/eye.2012.222

[3] Agrawal, A. and McKibbin, M. (2007) Purtscher's Retinopathy: Epidemiology, Clinical Features and Outcome. British Journal of Ophthalmology, 91, 1456-1459. https://doi.org/10.1136/bjo.2007.117408

[4] Massa, R., Vale, C., Macedo, M., et al. (2015) Purtscher-Like Retinopathy. Case Reports in Ophthalmological Medicine, 2015, Article ID: 421329. [PubMed]

[5] Blodi, B.A., Johnson, M.W., Gass, D.M., et al. (1990) Purtscher's-Like Retinopathy after Childbirth. Ophthalmology, 97, 1654-1659. [Google Scholar] https://doi.org/10.1016/S0161-6420(90)32365-5

[6] Olson, J., Rouhani, B. and Mandava, N. (2008) Sub-Tenon's Triamcinolone for Post Partum Purtscher's-Like Retinopathy. Clinical Ophthalmology, 2, 195-198. https://doi.org/10.2147/OPTH.S2080

[7] Meillier, A. and Rahimian, D. (2016) Cefepime-Induced Encephalopathy with Normal Renal Function. Case Reports, 6, 118-120. https://doi.org/10.1093/omcr/omw042

[8] Wu, C., Dai, R., Dong, F. and Wang, Q. (2014) Purtscher-Like Retinopathy in Systemic Lupus Erythematosus. American Journal of Ophthalmology, 158, 1335-1341. [PubMed] [Ref list]. https://doi.org/10.1016/j.ajo.2014.09.001 